\title{
Perceptually-Based Functions for Coarseness Textural Feature Representation
}

\author{
J. Chamorro-Martínez ${ }^{1}$, E. Galán-Perales ${ }^{1}$, B. Prados-Suárez ${ }^{2}$, \\ and J.M. Soto-Hidalgo ${ }^{1, \star}$ \\ ${ }^{1}$ Department of Computer Science and Artificial Intelligence, University of Granada \\ C/ Periodista Daniel Saucedo Aranda s/n, 18071 Granada, Spain \\ \{jesus, elena, soto\}@decsai.ugr.es \\ ${ }^{2}$ Department of Computer Science, University of Jaén \\ C/ Alfonso X el Sabio s/n, 23700 Linares, Jaén, Spain \\ belenps@ujaen.es
}

\begin{abstract}
Coarseness is a very important textural concept that has been widely analyzed in computer vision for years. However, a model which allows to represent different perception degrees of this textural concept in the same way that humans perceive texture is needed. In this paper we propose a model that associates computational measures to human perception by learning an appropriate function. To do it, different measures representative of coarseness are chosen and subjects assessments are collected and aggregated. Finally, a function that relates these data is fitted.
\end{abstract}

Keywords: Image features, textural features, human perception, visual coarseness.

\section{Introduction}

Texture is being increasingly recognized as an important cue for the analysis of natural imagery. Great research efforts are devoted to analyze this visual feature mainly due to the imprecission relative to its concept. In fact, different definitions of texture are found over the literature being described by some authors as local changes in the intensity patterns while others consider texture as a set of basic items or texels set out in conformity with some rules. Moreover, texture is also described by humans according to some concepts like coarseness, regularity, orientation [1].

Different approaches for texture characterization have been developed over the years. Haralick [2] proposed a classification of such approaches in two main groups: statistical methods (which analyze gray tone spatial distribution by computing some statistics on pixels intensity) and structural methods (which characterize texture by means of texels arranged in a certain way that is given by a set of rules). The author used statistics of Gray Level Coocurrence Matrices as

\footnotetext{
* This work has been supported by the MEC under the TEC2006-13845 project. 

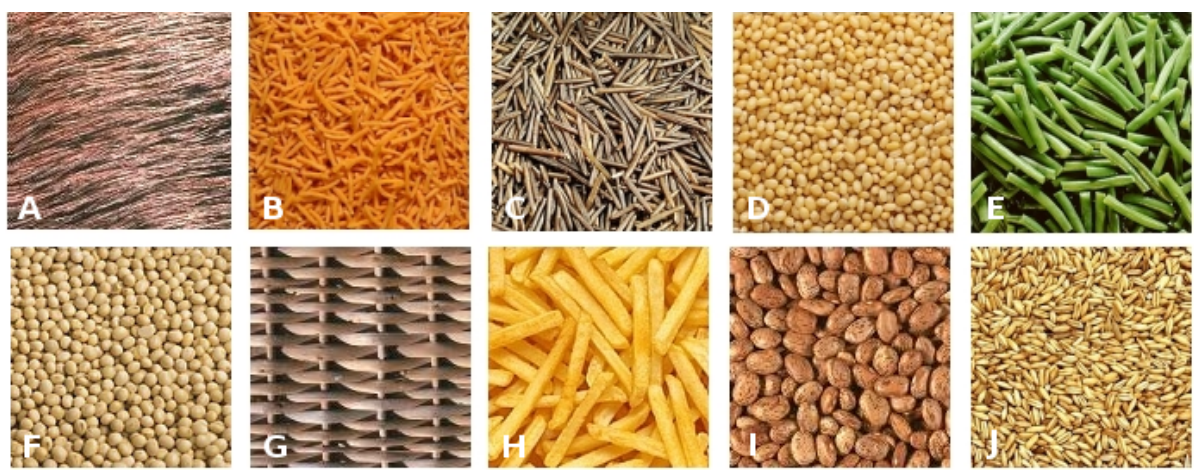

Fig. 1. Some examples of images with different degrees of fineness

measures of texture but such statistical values do not consider the way humans perceive texture. One of the first papers where textural measures are used taking into account human perception of texture was presented by Tamura [3. However, in Tamura's paper the relationship between the computational measures and the human perception of the different textural features is not learnt, just a rank correlation value is given. This value is calculated from the ordering results obtained from humans and from the computational measures of a small set of images. More recent approaches perform experiments with humans in order to model human perception but it is frequently found that the results given by such models can just compare two images and give a measure of their similarity [45] or they just analyze the presence or not of texture [6] but the presence degree of texture for a given image is not obtained.

In this paper we focus our study on coarseness, one of the textural properties most used in the literature which allows to distinguish between fine and coarse textures. In fact, the concept of texture is usually associated to the presence of fineness. A fine texture can be considered as small texture primitives with big gray tone differences between neighbour primitives (e.g. the image in figure 1(A)). On the contrary, if texture primitives are bigger and formed by several pixels, it is a coarse texture (e.g. the image in figure 1(I)).

In our approach we propose to model fineness by learning a function that captures human perception. To do this, two questions will be faced: what data should be used as independent variable, and how to obtain the related function. To solve the first question, a set of measures will be automatically computed from the texture image. To answer the second question, functional relationship between a certain measure and the presence degree of a textural concept related to it will be learnt.

The rest of the paper is organized as follows. In section 2 we introduce our methodology to obtain the functions that model the fineness textural concept. In section 3 we show the results of applying the models and the main conclusions and future work are sumarized in section 4 . 


\section{Obtaining Functions for Fineness Representation}

There are different measures over the literature that, given an image, capture the fineness (or coarseness) presence in the sense that the greater the value given by the measure, the greater the perception of texture. However two main drawbacks are found: on the one hand, there is no perceptual relationship between that value and the degree that humans perceive the texture; on the other hand, there are no thresholds that, given a certain measure, allow to decide whether there is fine texture, coarse texture or something intermediate (i.e. there are no intervals on the measure domain allowing for textural interpretation).

In this paper, we face these questions by proposing a function of fineness perception defined on the domain of a given measure. Concretely, we propose to model the fineness perception by means of a function

$$
\mathcal{T}: \mathbb{R} \rightarrow[0,1]
$$

defined as 1

$$
\mathcal{T}\left(x ; a_{n} \ldots a_{0}, \alpha, \beta\right)= \begin{cases}0 & x<\alpha, \\ \operatorname{poly}^{n}\left(x ; a_{n} \ldots a_{0}\right) & \alpha \leq x \leq \beta, \\ 1 & x>\beta\end{cases}
$$

with $\operatorname{poly}^{n}\left(x ; a_{n} \ldots a_{0}\right)$ being a polynomial function of the form:

$$
\operatorname{poly}^{n}\left(x ; a_{n} \ldots a_{0}\right)=a_{n} x^{n}+a_{n-1} x^{n-1}+\ldots+a_{1} x^{1}+a_{0}
$$

The value given by the function $\mathcal{T}$ will indicate how fine or coarse is the texture present in an image. Thus, a value of 1 will mean fineness presence while a value of 0 will mean no fineness presence (i.e. coarseness presence). Moreover, the parameters $\alpha$ and $\beta$ will split the domain of the measure in three intervals: $(-\infty, \alpha)$ corresponding to values related to coarse textures, $(\beta, \infty)$ corresponding to values related to fine textures, and $[\alpha, \beta]$ corresponding to intermediate values. For values in the interval $[\alpha, \beta]$, the function defined in equation 2 will obtain a value between 0 and 1 representing the degree of how fine the image is perceived (the closer the value to 1 , the greater the perception of fineness).

In this paper, we propose to obtain the parameters that define the function given in equation 2 by "learning" a functional relationship between a certain measure and the perception degree of fineness. To learn this relationship, we will use a set $\mathcal{I}=\left\{I_{1}, \ldots, I_{N}\right\}$ of $N$ images that fully represent the perception of fineness. Also, a set of measures $\mathcal{P}=\left\{P_{1}, \ldots, P_{K}\right\}$ will be considered, with $P_{k} \in \mathcal{P}$ being a measure of the perception of fineness in an image (e.g. $\mathcal{P}=\{$ EdgeDensity, Variance, Entropy $\}$ ). Thus, for each image $I_{i} \in \mathcal{I}$, we will have (a) a value obtained applying the measure $P_{k} \in \mathcal{P}$ to the image $I_{i}$, noted as $m_{k}^{i}$, and (b) an assessment $v^{i}$ of the perception degree of fineness. To get

\footnotetext{
${ }^{1}$ Note that this function is defined for measures that increases according to the perception of fineness but for those that decreases, the function needs to be changed appropriately.
} 
this assessment, a poll will be performed (section 2.1). Thus, for a given measure $P_{k} \in \mathcal{P}$, the multiset $\Psi_{k}=\left\{\left(m_{k}^{1}, v^{1}\right), \ldots,\left(m_{k}^{N}, v^{N}\right)\right\}$ is obtained and the function $\mathcal{T}$ is estimated by carrying out a Least of Squares Fitting (section 2.2).

\subsection{Assessment Collection}

In this section, the way to obtain a vector $\Gamma=\left[v^{1}, \ldots, v^{N}\right]$ of the assessments of the perception degree of fineness from the image set $\mathcal{I}=\left\{I_{1}, \ldots, I_{N}\right\}$ will be described. Thus, firstly a criterion for choosing the image set $\mathcal{I}$ is needed. After that, a poll which allows to get assessments of the perception degree of fineness will be designed. These assessments will be obtained for each image in $\mathcal{I}$, so an aggregation of the different assessments will be performed.

The Texture Image Set. A set $\mathcal{I}=\left\{I_{1}, \ldots, I_{N}\right\}$ of $N=80$ images representative of the concept of fineness has been selected. Figure 1 shows some images extracted from the set $\mathcal{I}$. The selection was done to cover the different perception degrees of fineness with a representative number of images. Furthermore, the images have been chosen so that as far as possible, just one perception degree of fineness is perceived.

The Poll. Given the image set $\mathcal{I}$, the next step is to obtain assessments about the perception of fineness from a set of subjects. From now on we shall denote $\Theta^{i}=\left[o_{1}^{i}, \ldots, o_{L}^{i}\right]$ the vector of assessments obtained from $L$ subjects for image $I_{i}$. To get $\Theta^{i}$, subjects will be asked to assign images to classes, so that each class has associated a perception degree of texture. In particular, 20 subjects have participated in the poll and 9 classes have been considered. The first nine images in figure 1 show the nine representative images for each class used in this poll. It should be noticed that the images are decreasingly ordered according to the perception degree of fineness.

Assessment Aggregation. Our aim at this point is to obtain, for each image $I_{i} \in \mathcal{I}$, one assessment $v^{i}$ that summarizes the vector of assessments $\Theta^{i}$ given by the different subjects about the perception degree of fineness. To aggregate opinions we have used an OWA operator guided by the quantifier "the most" [7] which allows to represent the opinion of majority of the polled subjects.

\section{$2.2 \quad$ Fitting the Function}

At this point, the aim is to obtain the function $\mathcal{T}$ (equation 2) from the multiset $\Psi_{k}$ corresponding to each measure $P_{k} \in \mathcal{P}$. In this paper, this function is calculated by carrying out a Least Squares Fitting taking into account the restriction of obtaining a monotonic function and considering $\mathrm{n}=1,2,3$ (i.e. linear, cuadratic and cubic functions) to define the polynomial function. In this paper, we have selected as $P$ a wide variety of measures, up to 18 shown in the first column of table1(that includes classical statistical measures well known in the literature, measures in the frequency domain, etc.). 
Table 1. Fitting RMSE and parameter values that define the function related to each measure

\begin{tabular}{|l|c|c|c|c|c|c|c|}
\hline \multicolumn{2}{|c|}{} & \multicolumn{3}{|c|}{ Parameter values } & \multicolumn{2}{c|}{ Knots } \\
\hline Measures & RMSE & $a_{3}$ & $a_{2}$ & $a_{1}$ & $a_{0}$ & $\alpha$ & $\beta$ \\
\hline Tamura [3] & 0.2240 & - & -0.247 & 0.822 & 0.421 & 3.77 & 2.31 \\
Fractal dimension [8] & 0.2243 & - & - & 1.05 & -2.36 & 2.25 & 3.2 \\
Edge density [9] & 0.2483 & 562 & -536 & 175 & -19 & 0.25 & 0.387 \\
Amadasun [10] & 0.2546 & -0.0007 & 0.0282 & -0.376 & 2.22 & 20.3 & 4.66 \\
Correlation [2] & 0.2555 & - & - & -1.15 & 1.12 & 0.978 & 0.106 \\
Local Homogeneity [2] & 0.3022 & -953 & 395 & -54.7 & 2.9 & 0.211 & 0.0514 \\
SNE [1] & 0.3065 & 51.5 & -110 & 77.8 & -18 & 0.511 & 0.935 \\
Weszka [12] & 0.3159 & 106 & -42.7 & 9.87 & -0.132 & 0.0142 & 0.203 \\
Newsam [3] & 0.3179 & 0.0306 & -1.71 & 31.9 & -198 & 16.2 & 21.3 \\
Wu 14] & 0.3186 & $6 \cdot 10^{-6}$ & -0.0006 & 0.0422 & -0.119 & 2.95 & 42.7 \\
Short Run Emphasis [15] & 0.3349 & 17900 & -52000 & 50300 & -16200 & 0.94 & 1 \\
Contrast [2] & 0.3459 & $9 \cdot 10^{-13}$ & $-3 \cdot 10^{-8}$ & 0.0003 & -0.024 & 70.6 & 5420 \\
Entropy [2] & 0.3622 & 1.22 & -31.6 & 273 & -785 & 8.02 & 9.44 \\
Uniformity[2] & 0.3802 & $-5 \cdot 10^{10}$ & $5 \cdot 10^{7}$ & -17900 & 2.19 & 0.0005 & $9 \cdot 10^{-5}$ \\
Variance[2] & 0.3987 & - & - & $5 \cdot 10^{-5}$ & -0.267 & 5880 & 27800 \\
DGD [16] & 0.4073 & 0.0001 & -0.0104 & 0.205 & -0.194 & 1 & 14.1 \\
FMPS [17] & 0.4127 & 0.005 & -0.081 & -1.62 & 27.2 & 18.2 & 19.9 \\
Abbadeni [1] & 0.4928 & - & - & 98.5 & -49.4 & 0.502 & 0.512 \\
\hline
\end{tabular}

Table 1 shows for each measure $P_{k} \in \mathcal{P}$ the least RMSE fitting error obtained, the corresponding parameters $\left(a_{3}, \ldots, a_{0}\right)$ and the knot values $\alpha$ and $\beta$ necessary to define the spline function. Note that the fitting error can be viewed as a goodness measure of the ability of the measure to represent the perception of fineness. Table 1 has been sorted in increasing order of the errors and it can be noticed that the five first measures obtain a similar RMSE which difference is as much of 0.03 what implies that these measures will give similar results when employed. Furthermore, the last measures appearing in table 1 have associated a high RMSE very distant from the above mentioned measures which implies that little representativeness of the perception of fineness is found for those measures.

\section{Results}

The function $\mathcal{T}$ obtained for each measure (defined by the parameter values shown in table 1) has been applied to different real images. Table 2 shows three real images with different perception degree of fineness. For each image and each measure, this table shows the value obtained by the related function and the error obtained when comparing this value with the assessment value given by subjects (by computing the difference between both of them). It can be noticed by looking the results shown in this table that our model allows to represent appropriately the perception of fineness. Note that the values given by the different functions are similar to the corresponding assessment degree for most of the obtained functions. 
Table 2. Estimated and error values obtained by applying the proposed model to three real images

\begin{tabular}{|c|c|c|c|c|c|c|}
\hline \multirow[b]{2}{*}{ Measure } & \multicolumn{2}{|c|}{$\begin{array}{c}\text { Assessment } \\
\text { Value }=0\end{array}$} & \multicolumn{2}{|c|}{$\begin{array}{c}\text { Assessment } \\
\text { Value }=0.5\end{array}$} & \multicolumn{2}{|c|}{$\begin{array}{c}\text { Assessment } \\
\text { Value }=1\end{array}$} \\
\hline & \begin{tabular}{|l|l|} 
Value \\
\end{tabular} & Error & Value & Error & Value & Error \\
\hline Tamura & \begin{tabular}{|l|}
0 \\
\end{tabular} & 0 & 0.545 & 0.045 & 0.966 & 0.034 \\
\hline Fractal dimension & 0 & 0 & 0.594 & 0.094 & 1 & 0 \\
\hline Edge density & 0 & 0 & 0.488 & 0.012 & 1 & 0 \\
\hline Amadasun & 0 & 0 & 0.489 & 0.011 & 0.854 & 0.146 \\
\hline Correlation & 0.012 & 0.012 & 0.475 & 0.025 & 1 & 0 \\
\hline Local Homogeneity & 0 & 0 & 0.459 & 0.041 & 0.735 & 0.265 \\
\hline SNE & 0 & 0 & 0.421 & 0.079 & 0.843 & 0.157 \\
\hline Weszka & 0.085 & 0.085 & 0.572 & 0.072 & 0.741 & 0.259 \\
\hline Newsam & 0 & 0 & 0.435 & 0.065 & 0.605 & 0.395 \\
\hline $\mathrm{Wu}$ & 0.076 & 0.076 & 0.607 & 0.107 & 0.774 & 0.226 \\
\hline Short Run Emphasis & 0 & 0 & 0.548 & 0.048 & 0.709 & 0.291 \\
\hline Contrast & 0.028 & 0.028 & 0.634 & 0.134 & 0.604 & 0.396 \\
\hline Entropy & 0.285 & 0.285 & 0.687 & 0.187 & 0.484 & 0.516 \\
\hline Uniformity & 0.115 & 0.115 & 0.820 & 0.320 & 0.663 & 0.337 \\
\hline Variance & 0.271 & 0.271 & 0.640 & 0.140 & 0.743 & 0.257 \\
\hline DGD & 0 & 0 & 0.331 & 0.169 & 1 & 0 \\
\hline FMPS & 0.360 & 0.360 & 0.717 & 0.217 & 0.803 & 0.197 \\
\hline Abbadeni & 0.396 & 0.396 & 0.396 & 0.104 & 0.396 & 0.604 \\
\hline
\end{tabular}

Let's consider figure 2(A) corresponding to a mosaic made by several images, each one with a different increasing perception degree of fineness. The perception degree of fineness for each subimage has been calculated using the Tamura measure (the one with least fitting error according to table 1) and the results are shown in figure 2(B) where a white grey level means maximum perception of fineness, while a black one corresponds to no perception of fineness (the numeric value is also shown on each subimage). It can be noticed that our model captures the evolution of the perception degrees of fineness.

Table 3 shows a comparative between our model and the assessments obtained from subjects for the images in figure 2 The second column shows the assessments given by humans for the different images. The third column shows the perception degree of fineness obtained by applying our model, the fourth column shows the difference between the computed degree and the human assessment. In the case of the fifth column we calculate the differences between the assessment given by each subject and the computed degree, and we obtain 

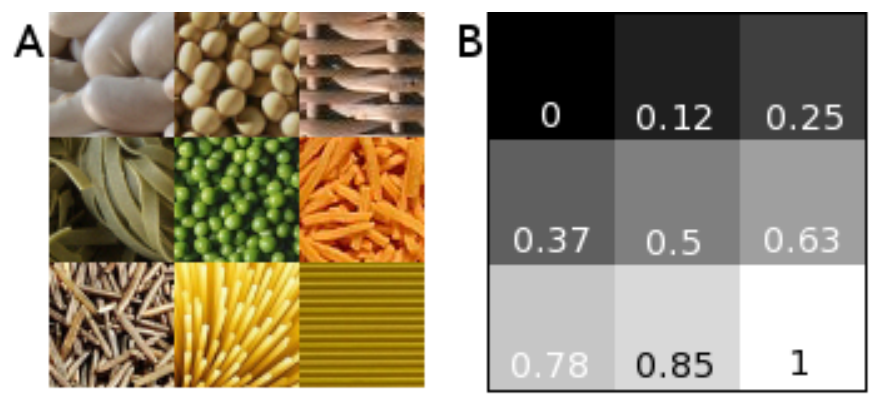

Fig. 2. Results for a mosaic image: (A) original mosaic image (B) presence degree of fineness textural concept obtained with the proposed model for the Tamura measure

Table 3. Errors obtained from mosaic image of figure 2 for the Tamura measure

\begin{tabular}{|c|c|c|c|c|}
\hline Image & $\begin{array}{c}\text { Human } \\
\text { Assessment }(\mathrm{H})\end{array}$ & \begin{tabular}{|l|} 
Estimated \\
Value $(\mathrm{V})$
\end{tabular} & $\begin{array}{l}\text { Error \#1 } \\
(|H-V|)\end{array}$ & Error \#2 \\
\hline 1 & 0 & 0 & 0 & 0 \\
\hline 2 & 0.125 & 0.125 & 0 & 0.021 \\
\hline 3 & 0.250 & 0.250 & 0 & 0.010 \\
\hline 4 & 0.375 & 0.375 & 0 & 0.094 \\
\hline 5 & 0.500 & 0.500 & 0 & 0.010 \\
\hline 6 & 0.625 & 0.625 & 0 & 0.052 \\
\hline 7 & 0.750 & 0.780 & 0.030 & 0.010 \\
\hline 8 & 0.875 & 0.852 & 0.023 & 0.010 \\
\hline 9 & 0.960 & 1 & 0.040 & 0.052 \\
\hline & & & Avg: 0.011 & Avg: 0.029 \\
\hline
\end{tabular}

as error measure the mean from these 20 differences. Finally, the average errors shown in the last row with values of 0.011 and 0.029 show the goodness of our approach to represent the subjectivity found in fineness perception.

\section{Conclusions and Future Works}

In this paper, functions of fineness perception have been defined on the domain of certain measures. To define such functions, the relationship between a given measure (automatically computed over the image) and the perception degree of fineness has been achieved. In order to obtain the perception degree of fineness, a group of human subjects have been polled. The functions presented in this paper allow to decide whether there is fine, coarse or intermediate texture, splitting the domain into three perceptual meaningful intervals. The results given by our approach show a high level of connection with the assessments given by subjects.

As future work, we will extend the proposed approach to obtain functions on $\mathbb{R}^{n}$, i.e. functions on vectors of measures. Furthermore, the performance of the fineness functions will be analyzed in applications like textural classification or segmentation. 


\section{References}

1. Abbadeni, N., Ziou, N., Wang, D.: Autocovariance-based perceptual textural features corresponding to human visual perception. In: Proc. of 15th International Conference on Pattern Recognition. Volume 3. (2000) 901-904

2. Haralick, R.: Statistical and structural approaches to texture. Proceedings IEEE 67(5) (1979) 786-804

3. Tamura, H., Mori, S., Yamawaki, T.: Textural features corresponding to visual perception. IEEE Trans. on Systems, Man and Cybernetics 8 (1978) 460-473

4. Fahmy, G., Black, J., Panchanathan, S.: Texture characterization for joint compression and classification based on human perception in the wavelet domain. IEEE Transactions on Image Processing (2006)

5. Manian, V., Vasquez, R.: Texture discrimination based on neural dynamics of visual perception. In: Proc. International Joint Conference on Neural Networks. Volume 1. (2003) 113-118

6. Chen, J., Pappas, T., Mojsilovic, A., Rogowitz, B.: Adaptive perceptual colortexture image segmentation. IEEE Transactions on Image Processing (2005)

7. Yager, R.: On ordered weighted averaging aggregation operators in multicriteria decisionmaking. IEEE Trans. on SMC 18(1) (1988) 183-190

8. Peleg, S., Naor, J., Hartley, R., Avnir, D.: Multiple resolution texture analysis and classification. IEEE Transactions on Pattern Analysis and Machine Intelligence (4) (1984) 518-523

9. Canny, J.: A computational approach to edge detection. IEEE Transactions on Pattern Analysis and Machine Intelligence 8(6) (1986) 679-698

10. Amadasun, M., King, R.: Textural features corresponding to textural properties. IEEE Transactions on Systems, Man and Cybernetics 19(5) (1989) 1264-1274

11. Sun, C., Wee, W.: Neighboring gray level dependence matrix for texture classification. Computer Vision, Graphics and Image Processing 23 (1983) 341-352

12. Weszka, J., Dyer, C., Rosenfeld, A.: A comparative study of texture measures for terrain classification. IEEE Trans. on SMC 6 (1976) 269-285

13. Newsam, S., Kammath, C.: Retrieval using texture features in high resolution multi-spectral satellite imagery. In: Data Mining and Knowledge Discovery: Theory, Tools, and Technology VI, SPIE Defense and Security. (2004)

14. Wu, C., Chen, Y.: Statistical feature matrix for texture analysis. CVGIP: Graphical Models and Image Processing 54(5) (1992) 407-419

15. Galloway, M.: Texture analysis using gray level run lengths. Computer Graphics and Image Processing 4 (1975) 172-179

16. Kim, S., Choi, K., Lee, D.: Texture classification using run difference matrix. In: Proc. of IEEE 1991 Ultrasonics Symposium. Volume 2. (1991) 1097-1100

17. Yoshida, H., Casalino, D., Keserci, B., Coskun, A., Ozturk, O., Savranlar, A.: Wavelet-packet-based texture analysis for differentiation between benign and malignant liver tumours in ultrasound images. Physics in Medicine and Biology 48 (2003) 3735-3753 